\title{
Integrated Computer Control System Architectural Overview
}

\author{
P. Van Arsdall
}

June 18, 1997

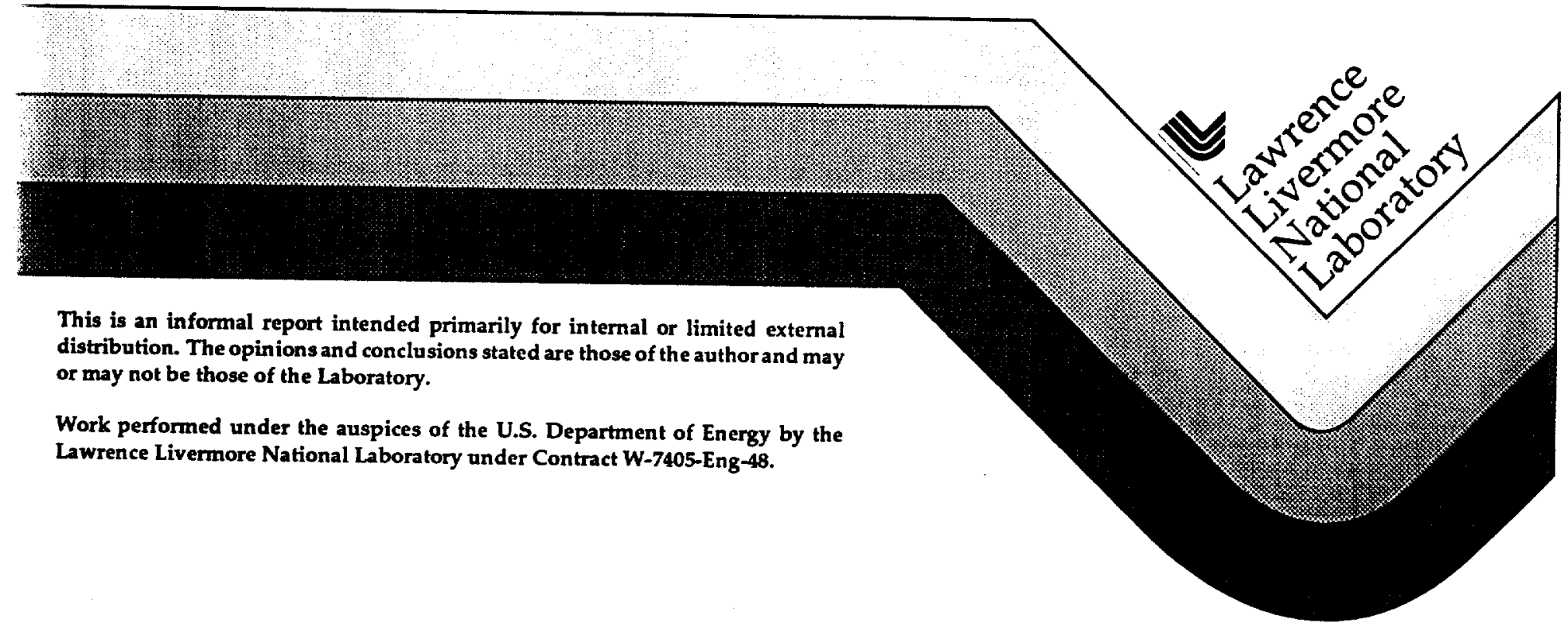




\section{DISCLAIMER}

This document was prepared as an account of work sponsored by an agency of the United States Government. Neither the United States Government nor the University of California nor any of their employees, makes any warranty, express or implied, or assumes any legal liability or responsibility for the accuracy, completeness, or usefulness of any information, apparatus, product, or process disclosed, or represents that its use would not infringe privately owned rights. Reference herein to any specific commercial product, process, or service by trade name, trademark, manufacturer, or otherwise, does not necessarily constitute or imply its endorsement, recommendation, or favoring by the United States Government or the University of California. The views and opinions of authors expressed herein do not necessarily state or reflect those of the United States Government or the University of California, and shall not be used for advertising or product endorsement purposes.

This report has been reproduced directly from the best available copy.

Available to DOE and DOE contractors from the Office of Scientific and Technical Information

P.O. Box 62, Oak Ridge, TN 37831

Prices available from (615) 576-8401, FTS 626-8401

Available to the public from the

National Technical Information Service

U.S. Department of Commerce

5285 Port Royal Rd.,

Springfield, VA 22161 
Architectural Overview

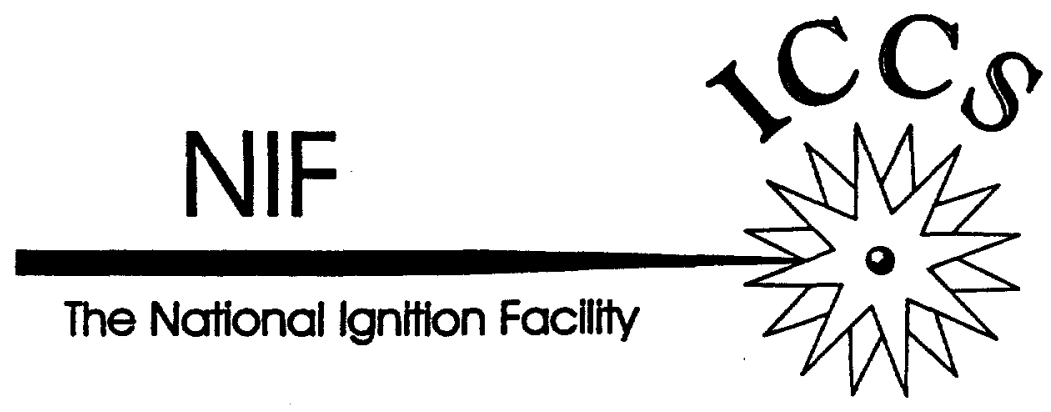




\section{Introduction}

This overview introduces the NIF Integrated Control System (ICCS) architecture. The design is abstract to allow the construction of many similar applications from a common framework. This summary lays the essential foundation for understanding the model-based engineering approach used to execute the design.

The NIF design effort is motivated by the magnitude of the task. The NIF requires integration of about 40,000 atypical control points, must be highly automated and robust, and will operate continuously around the clock. The risk associated with complex control systems can be great, and there have been recent examples where the utility of scientific facilities has been limited by inflexible system architectures. Typically control systems must absorb significant changes in requirements late in project construction, resulting from delayed requirement resolution in other areas. Furthermore, facilities such as the NIF represent major capital investments that will be operated, maintained, and upgraded for decades.

Risks to the infrastructure are mitigated in the ICCS design by incorporating modularity, segmentation, and open-systems standards so that components and subsystems can be replaced at designated interface points if necessary. Risks to the control system software are managed through a modern object-oriented software framework that is used to construct all applications and that will be extensible and maintainable throughout the project life cycle. This framework is reusable across multiple applications (and indeed other projects) and offers interoperability among computers and operating systems by leveraging a common object request broker architecture (CORBA).

The Integrated Computer Control System (ICCS) architecture was created to address the general problem of providing distributed controls for medium to large-scale scientific (or other: automated facilities) that do not require significant real-time capability within the supervisory software. The ICCS architecture is generally applicable to the class of event-driven control systems where client-server tactics are appropriate. This is the case for the NIF, as the shot time-line occurs over several hours and can be suspended if necessary. Some real-time control is inevitably necessary; this is handled either by the Integrated Timing System, the Industrial Control System segment or at the edges of the architecture. This overview is limited to discussing the client-server segment of the architecture that contains the supervisory software. Many terms used in this document are defined in reference 1.

The following sections discuss several important views of the system:

- Layered control system model

- NIF software applications

- Hardware infrastructure

- CORBA distribution

- Software development environment 
- Supervisory software framework

- Software deployment

\section{Layered Control System Model}

The ICCS is a layered architecture consisting of front end processors (FEP) coordinated by a supervisory system [Figure 1]. The supervisory layer, which is hosted on UNIX workstations, provides centralized operator controls and status, data archiving, and integration services. FEP units are constructed from VME-bus or Compact PCI crates of embedded controllers and interfaces that attach to control points. FEP software provides the distributed services needed to operate the control points by the supervisory system. Functions requiring real-time implementation are allocated to software within the FEP or embedded controller, which is located at the edge of the architecture and therefore does not require communication over the local area network. The software is distributed among the computers and provides plug-in software extensibility by using services from CORBA.

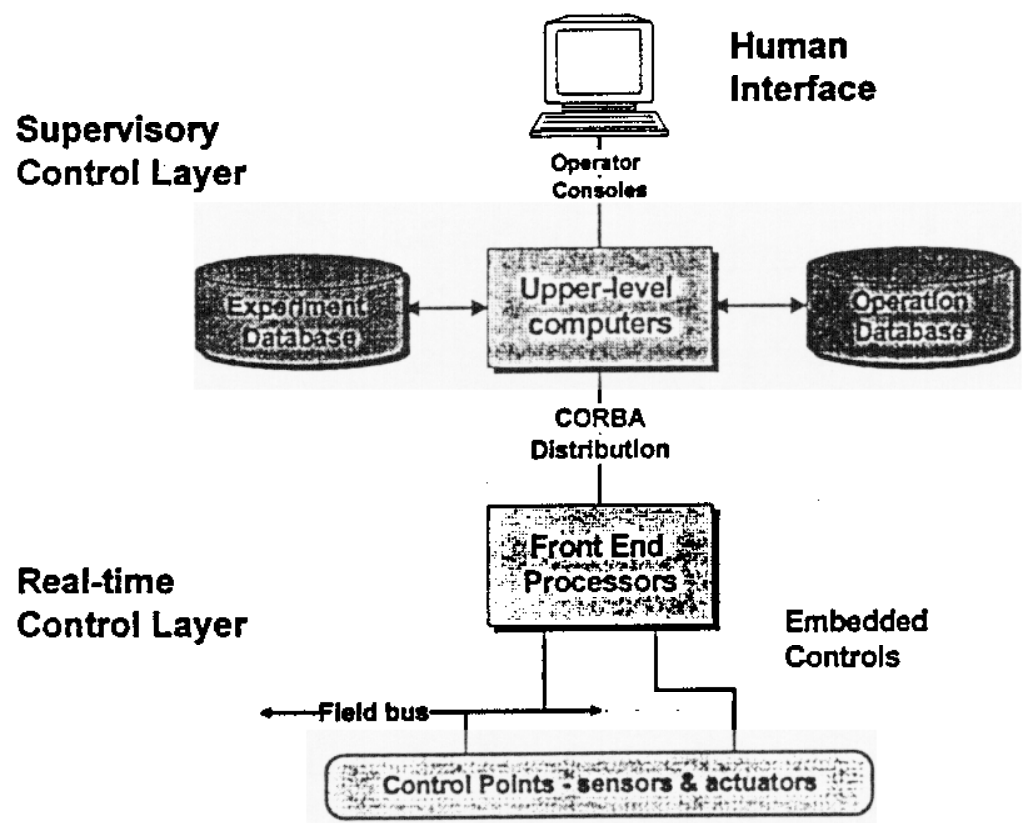

Figure $1 \quad$ Layered control system model

The supervisory control layer is a large software system that provides the human interface in the form of operator displays, data retrieval and processing, and coordination of control functions for the NIF. This layer is partitioned into several cohesive subsystems, each of which controls a primary NIF subsystem such as alignment or power conditioning. Several databases are incorporated to manage both experimental data and data used during operations and maintenance. The subsystems are integrated to coordinate operation of laser and target area equipment. The supervisory software is responsible for duties ranging from configuration and control sequencing to data processing and archival.

The real-time control layer contains many kinds of FEP that implement the distributed portion of the ICCS by interfacing to the NIF control points (aka devices). The FEP software performs sequencing, data acquisition and reduction, and device control. The software framework 
includes a standard way for FEP units to be integrated into the supervisory system by providing a common distribution mechanism coupled with pattems for device configuration, control, and status monitoring functions.

The control points are sensors and actuators attached to interface boards plugged into the FEP backplane. In many cases, control points are handled by intelligent components that incorporate local microprocessors operated by small fixed programs (known as embedded controllers). In a few cases, remote devices attach to FEP units by utilizing a low-cost network of microcontrollers known as a field bus. The software running in the embedded controller does much of the low-level work that would otherwise be imposed on the FEP. Example components in this layer are stepping motor controllers, photodiode detectors, and power supply controls.

\section{The NIF Software Applications}

Eight supervisory and thirteen front end processor applications are used to implement the specific requirements of the NIF control system as shown in the layered diagram of Figure 2. This partitioning of applications is derived from the organization of the NIF equipment and associated work breakdown structure. The cohesiveness and coupling of the architecture is good because most communication occurs either within an application's tasks or vertically between layers while minimizing coupling between adjacent applications.

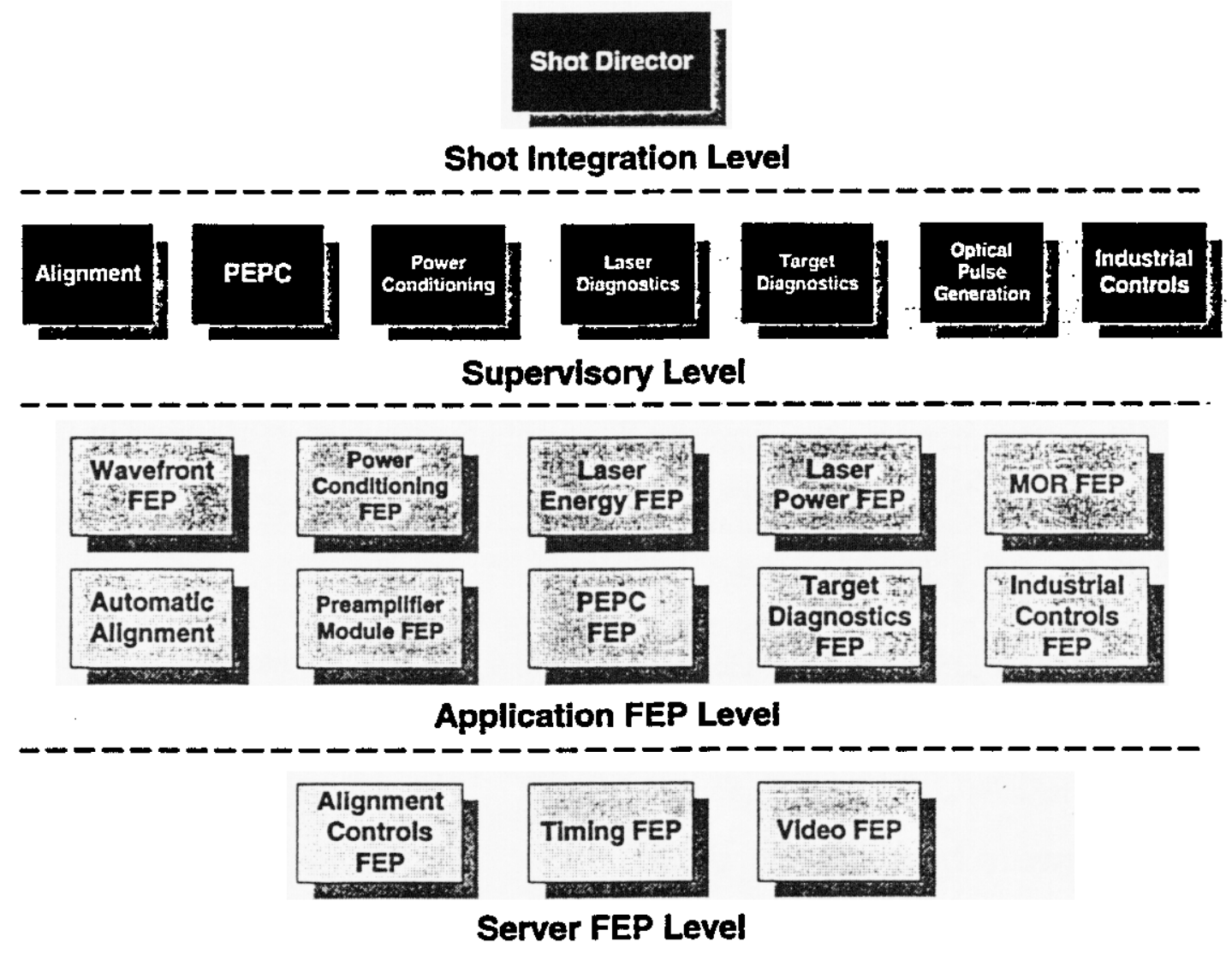

Figure 2 Software applications in the NIF control system 
At the topmost layer the Shot Director application coordinates the supervisory subsystems to achieve integrated facility operation. The bottom layer is comprised of FEPs that provide device control services to upper layer software. The Application FEP layer introduces capability for autonomous control and both provides services to upper layers and uses services from the layer below. The major control subsystems are represented in the Supervisory Layer, which are summarized below.

The Alignment application is an ensemble of processes that work in unison to provide manual and automatic alignment of the beam transport system. Integrating the largest of the subsystems, the software remotely controls and monitors thousands of motorized devices attached to over a hundred Alignment Control FEPs. Additional FEPs for Automatic Alignment and Wavefront control are integrated into this application.

The Plasma Electrode Pockels Cell (PEPC) application and FEP serves to control, diagnose, and maintain the electro-optic switch that enables multipass operation of the main laser amplifiers.

The Power Conditioning application integrates the control and monitoring of power supply modules and FEPs that are located in four high-voltage capacitor banks. Each module in turn drives and monitors multiple flash lamp circuits. Operators control the settings on the bank modules and view module status and feedback on graphical user interface (GUI) displays. The GUI monitors the charging voltage and lamp current during operation.

The Laser Diagnostics application and FEP measures laser performance and power on the target. Precision operation is achieved by adjusting the laser controls based on these measurements combined with a model of the laser chain performance. The subsystem integrates the control and collection of sensor energy data, laser pulse temporal data, and beam images from various locations on each of the beams.

The Target Diagnostic application and FEP is the most dynamic. The NIF is expected to accommodate a varying suite of diagnostics, and the control system provides an open architecture that defines high-level services that can be extended, with modest effort, to serve the needs of instruments that will arrive after the facility is operational.

Operation of the master oscillator and the 48 preamplifier modules is supervised by the Optical Pulse Generation (OPG) application and associated FEPs. This subsystem is particularly interesting because it contains independent elements of beam diagnostics, power conditioning, and alignment.

Industrial control rounds out the suite of supervisory applications by integrating the distributed portions of the separate Industrial Control segment, which is highlighted by the Safety Interlock System. This segment also includes the vacuum and gas controls in both the Beam Transport System and the Target Chamber System as well as incorporates the data logging performed by the Facility Environmental Monitor.

\section{Hardware Infrastructure}

Figure 3 shows the NIF computer system and network, which is comprised several hundred processors distributed over an area roughly two football fields in size. The main control room contains seven graphics consoles, each of which houses two workstations with dual displays. 
The applications discussed above are normally assigned to execute and be operated from one primary console, although the software can easily be moved and is intended to be operated from remote graphics terminals located near the front end equipment. A pair of file servers provides disk storage and archival databases for the entire system as well as well as hosting centralized management and naming services necessary for coordinating the facility operation.

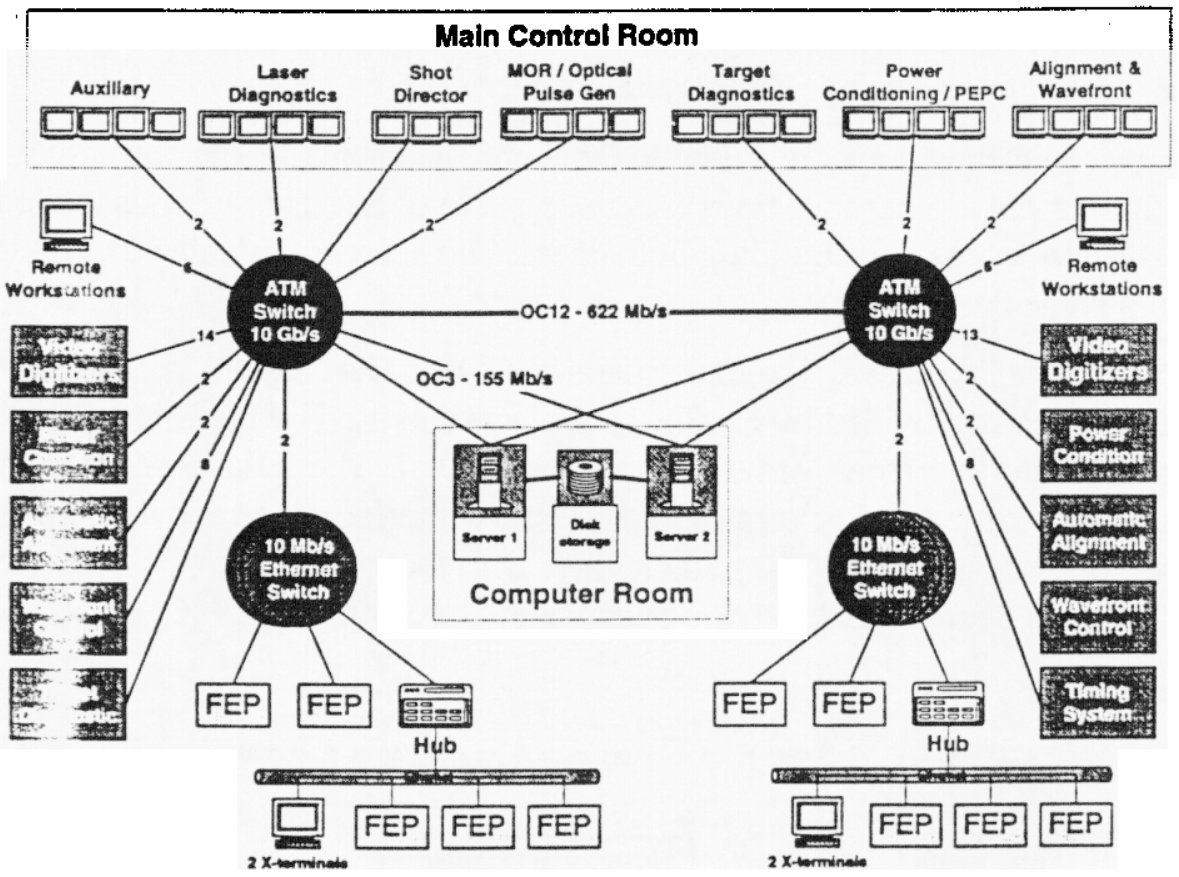

Figure 3 NIF computer system and network architecture

The netwo:- backbone is built from ATM switches that are capable of carrying digital motion video (iscc conous traffic) as well as TCP/IP (asynchronous traffic). TCP/IP is used for most. commun: .jons. FEPs are generally attached to shared Ethernet, switched Ethernet, or Fast Ethemet pending on the bandwidth requirements of the control devices and supervisory commur tion. Some FEPs and all workstations and file servers are attached directly to ATM for maxi. Im communications performance. ATM switching offers the advantage that the network . segmented to isolate traffic among groups of collaborating computers. Further: re, the ATM standard provides facilities (e.g. permanent virtual circuits and a quality ci service protocol) that guarantee bandwidth is available for time-critical commurications such as digital motion video.

\section{CORBA Distribution}

Past architectural approaches to distributed controls have relied on the technique of building large application programming interface (APD) libraries for each category. The API gives applications access to functions implemented throughout the architecture. This practice results in the programming of large numbers of application interconnections that quickly increase the system complexity, and make software maintenance and evolutionary change much more 
difficult. A more modem approach to the distributed computing problem is to use a clientserver architecture in which software processes communicate using CORBA distribution ${ }^{2}$.

CORBA is a standard developed by a consortium of all major computer vendors to propel the dominance of distributed objects on local area networks and the worldwide web. The best way to think of CORBA is as the universal "software bus". CORBA is a series of sophisticated, but standard sockets into which software objects can "plug and play" to interoperate with one another. Even when made by different vendors, at different times, the object interfaces are standard enough to coexist and interoperate. By design, CORBA objects operate across languages, operating systems, networks, and tools.

When objects interact it is convenient to label one the client (i.e. the object that initiates the interaction), and the other the server (i.e. the passive object that responds to the initiative). CORBA provides tools for building both clients and servers, and indeed allows an object to be a client in some interactions and a server in others.

At a greatly simplified level, the major parts of CORBA are shown in Figure 4. The interface types and methods provided by the Server Objects and used by the Clients are defined by an industry standard Interface Definition Language (IDL). The IDL compiler examines the interface specification and generates the necessary interface code and templates into which user-specific code is added. The code in the Client that makes use of CORBA objects is written as if the Server was locally available and directly callable - CORBA takes care of all the rest.

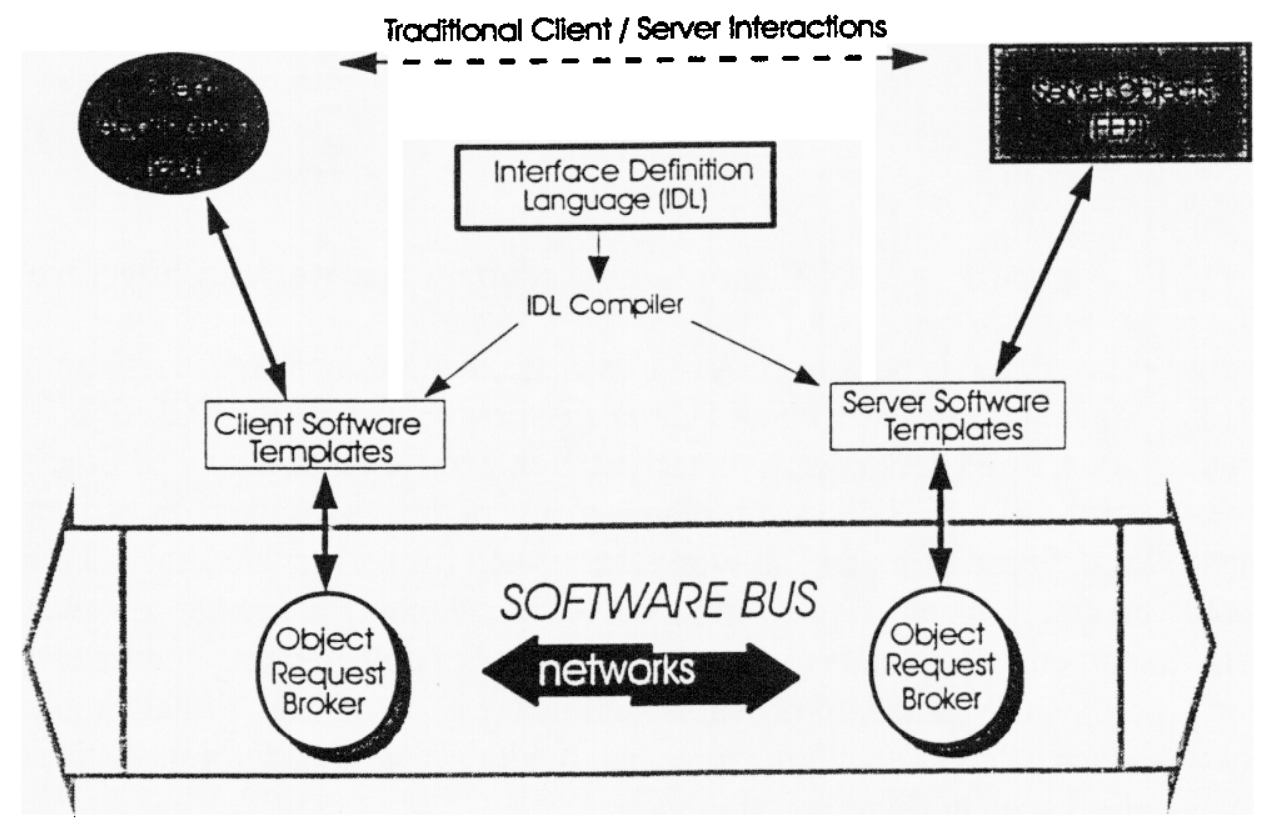

Figure 4 CORBA distribution implements a software bus on the network

\section{ICCS Software Development Environment}

ICCS software development is managed under a software quality assurance plan ${ }^{3}$ that covers the entire life cycle of the software design, production, and maintenance. Supporting that plan 
are software engineering tools that are proven capable of executing large object-oriented projects. Object-oriented analysis and design follow the strategies expressed by Booch ${ }^{4}$.

The tools and the integrated software development process are depicted in Figure 5. Central to the ICCS development are documentation standards for requirement specifications (SRS) and software design descriptions (SDD). These documents are essential to the long-term maintainability of the software in view of periodic software upgrades and staffing turnover expected over the 30-year life of the NIF.

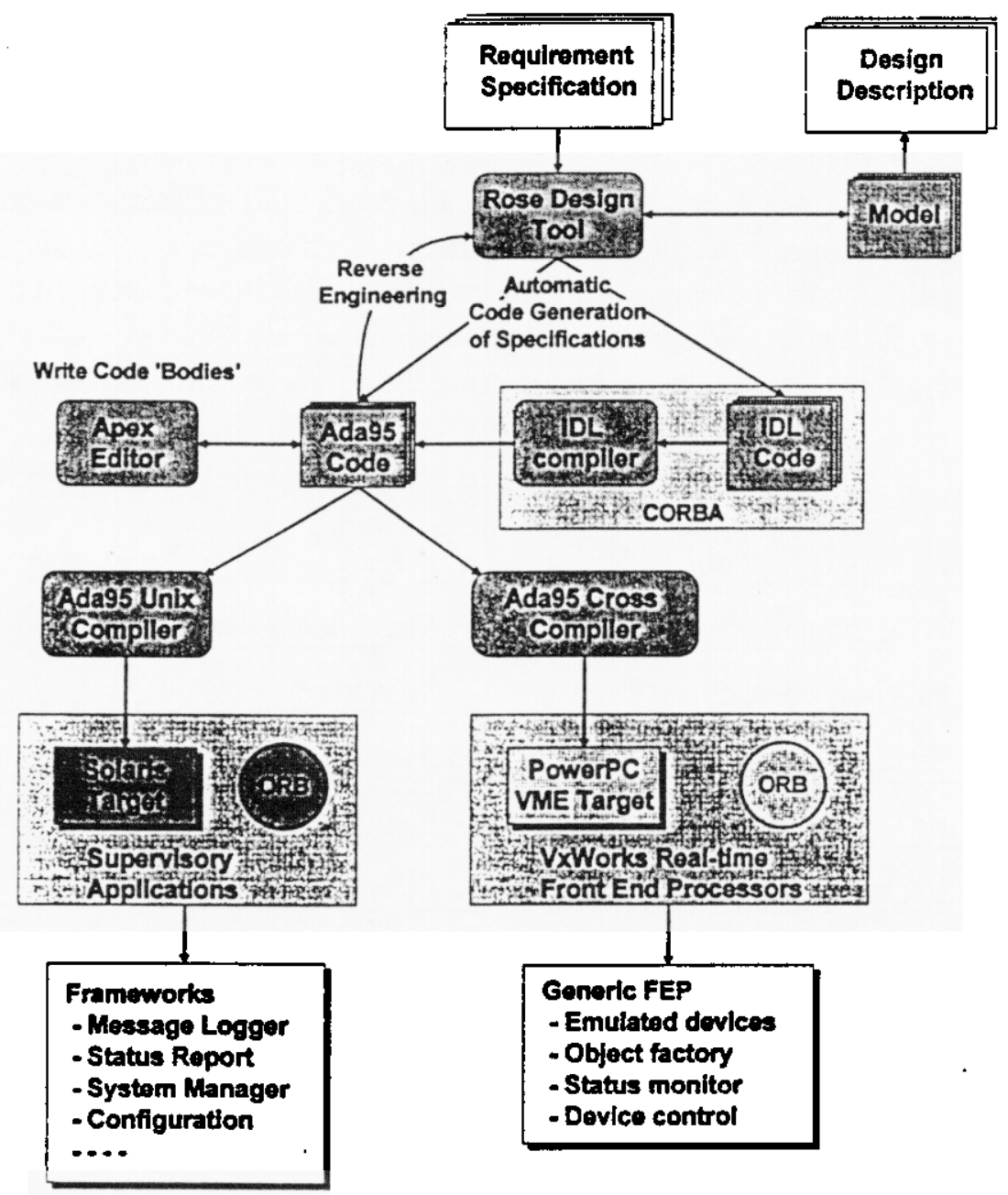

Figure 5 Flowchart of the ICCS software engineering process

Detailed requirements expressed in the SRS are analyzed and result in the object-oriented design maintained by the Rose tool. Classes are defined to implement the responsibilities of the software. Important software use cases are expressed in terms of object scenario diagrams. In general terms, Rose is used to model the interfaces and interactions between major software entities in the design. After the modeling cycle is complete, the Rose tool generates the Ada 
specifications corresponding to the class interface and type definitions. The developer fills in the detailed coding necessary to implement internal details of each class. Classes that are distributed generate IDL, which is passed through the IDL compiler to generate Ada code as before. The SDD is a narrative document for explaining the object-oriented model and contains other design material necessary for implementing the software.

The Ada source code then takes one of two paths depending on the target processor architecture. For Solaris, the source is compiled, linked, debugged, and tested on the workstation. For PowerPC or other VxWorks targets, the source is compiled and debugged under the Apex Works cross-compilation system. In either case, the models, sources, binaries, and run-time images are managed by the Apex configuration management system, which provides full capability for version control. Using Apex subsystem facilities, the frameworks can be independently developed by different engineers, each of which has a protected view of the other components.

The ICCS incorporates Ada, CORBA, and object-oriented techniques to enhance the openness of the architecture and portability of the software. As a consequence, many alternate implementation choices exist now and more will surface in the future. Notable examples of interesting tools include Windows NT and the Intel family of processors. At the current state of implementation, the tools used to construct the ICCS are indicated in Table 1.

Table 1 Tools used in the construction of the ICCS

\begin{tabular}{l|l}
\multicolumn{1}{c|}{ Tool } & \multicolumn{1}{c}{ Vendor \& type } \\
\hline UNIX OS and workstations & Sun Solaris 2.5 on Sparc and UltraSparc \\
\hline Real-time operating system & Windriver VxWorks 5.3 \\
\hline FEP CPU chipset & Motorola PowerPC family \\
\hline Ada 95 host compiler & Rational Ada (Solaris) \\
\hline Alternate Ada 95 host compiler & GNAT Ada (Solaris) \\
\hline Ada 95 cross compiler & Rational Apex Works (VxWorks) \\
\hline C++ host compiler & Rational C++ \\
\hline Configuration management & Rational Apex \\
\hline CORBA on UNIX and VxWorks & Objective Interface Systems Orbix/Ada \\
\hline Relational and object database & Oracle 8 \\
\hline Graphical user interface & UIM/Orbix \\
\hline Object-oriented modeling & Rational Rose / Ada
\end{tabular}




\section{ICCS Supervisory Software Framework}

The ICCS supervisory software framework is a collection of collaborating abstractions that are used for the construction of the application software. Frameworks ${ }^{5}$ reduce the amount of coding necessary by providing prebuilt components that can be extended to accommodate specific additional requirements. The framework also promotes code reuse by providing a standard model and interconnecting backplane that is shared from one application to the next. The framework concept enables the cost-effective construction of the NIF software and provides the basis for long-term maintainability and upgrades.

Components in the ICCS framework plug into the CORBA bus, as shown in Figure 6. Using this very simple view of the components, a few scenarios can be described. Graphical user interface works with status report to provide operator controls by collaborating with device control and status monitor objects located in the front end processor. Archive (and a commercial database) adds the services needed for experimental data processing and trending of machine performance. Configuration interacts with object factories to create, locate, and coordinate the software objects that implement device services within the FEP. Other components (not shown in the figure) provide event logging and process interlock services.

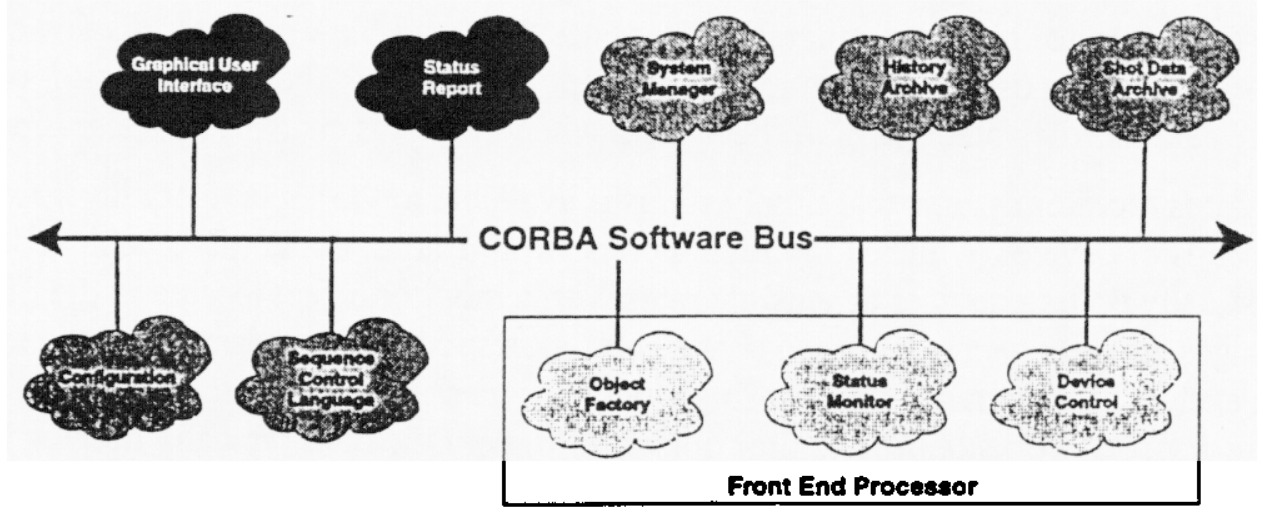

Figure 6 ICCS framework objects plug into the CORBA software bus

An example of two objects that collaborate is the interaction between a status monitor object that watches a motor device and informs a particular status report object when the motor moves. The status report (on the supervisory workstation) is responsible to collect the stream of status updates from the status monitor (on the FEP) and make them available to the operators. The status report object updates a graphic user interface so an operator can see the current status of the motor device. This scenario is an example of two objects that work together smoothly even though they are on different computers.

The following subsections introduce the ten frameworks that form the basis of the ICCS supervisory software.

\subsection{Configuration}

Configuration is a hierarchical organization for the static data that define the hardware control points that are accessible to the ICCS. Configuration provides a taxonomic system that is used as the key by which clients locate devices (and other software services) on the CORBA bus; 
the configuration hierarchy is the unique source of device identifying information within the ICCS. During normal operation, configuration provides to clients the CORBA references to all distributed objects. A useful feature of configuration is the ability to select entire branches of the hierarchy for subsequent operations.

The naming convention for NIF devices is described in reference 6. A taxon is the hierarchical name given to nodes in the configuration data structure and has the general form:

$$
\text { Taxon } \Rightarrow \text { (Location, Unit, Component, Identifier). }
$$

An important responsibility of configuration is the initialization of front end processors during startup. Configuration data are stored in the database and describe how and where the control hardware is installed in the system. Calibration data for sensors, setpoints for alignment devices, and VO channels used by devices on interface boards are examples of static data managed by configuration. During ICCS startup, this framework collaborates with an object factory located in the FEP. Using the data and methods stored in the configuration database, the object factory instantiates, initializes, and determines the CORBA reference for each device and controller object in the FEP.

\subsection{Status Monitor}

The status monitor provides generalized services for broad-view operator display of device status information using the push model of event notification. The status monitor observes devices and notifies other parts of the system when the status changes by a significant amount.

The status monitor assures that timely status is available to the supervisory layer from thousands of devices, while economizing on network traffic. Economy is achieved by periodically polling devices at sensible rates with a monitor object located in the FEP; network messages are only generated when changes of interest occur. Furthermore, status information from each FEP is aggregated into a single network message for transmission to the client in the supervisory workstation. Monitor objects have periodic rates typically ranging from 0.1 to 10 seconds. A filtering mechanism is provided for managing the change threshold.

\subsection{Sequence Control Language}

The sequence control language (SCL) framework is used to create custom scripting languages for the NIF applications. The SCL service automates sequences of commands executed on the distributed control points or other software artifacts. Each SCL instance provides access to a subset of the ICCS device control and status methods available for a particular application domain area. Script languages are implemented internally with the Tool Command Language (Tcl) interpreter.

The SCL provides the following control constructs:

- Serial execution of commands either inside or outside of control constructs

- Indeterminate loops (while-true-loop)

- Conditional branching (if-then-elsif-else-endif)

- Synchronized concurrent threads of control (fork-join)

- Passing of user-supplied arguments to user-supplied methods at run-time

- Dispatching call to user-supplied method 
Operators create and edit sequences by selecting icons that represent control constructs, boolean functions, and user-supplied methods from a visual programming palette. The icons are then interconnected to program the sequence and any boolean conditions or method arguments needed are defined to complete the sequence script. Examples include a checklist SCL for use by Laser Diagnostics operators and an embedded SCL for closed-loop processing in the Automatic Alignment FEP.

\subsection{Graphical User Interface}

All human interaction with the ICCS will be via graphical user interfaces displayed upon control room consoles or on X Terminals distributed throughout the facility. The GUI is implemented as a framework in order to ensure consistency across the applications. The GUI framework is based upon the X Windows system and Motif policies. Commercial GUI development tools are used to construct the display graphics. In addition, the color scheme used on Nova is incorporated to provide continuity to operators as they migrate from that system to the NIF.

This framework consists of guidelines for look and feel as well as some common graphical elements:

- NIF Control Panel

- Beam Selector

- Laser Map

- Status Summary

- Countdown clock

An example of one of the common graphical elements is the top-level NIF Control Panel shown in Figure 7.

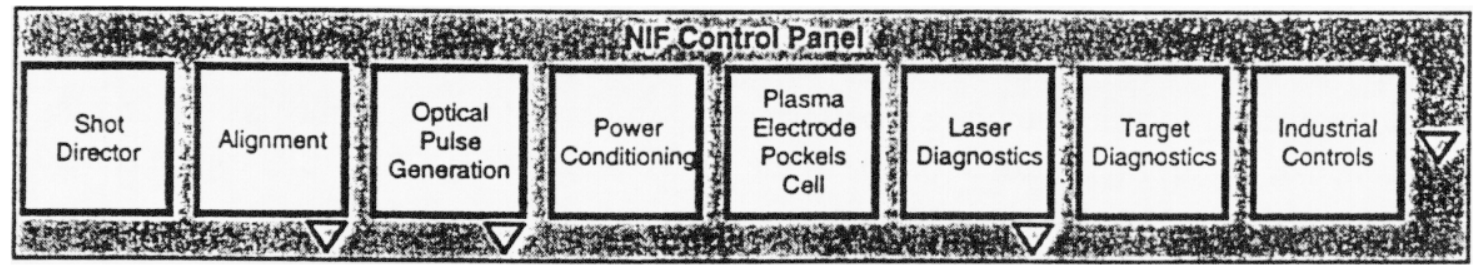

Figure 7 GUI panel used to access all NIF subsystem interfaces.

\subsection{Message Log}

The message log provides event notification and archiving services to all subsystems or clients within the ICCS. A central server collects incoming messages and associated attributes from processes on the network, writes them to appropriate persistent stores, and also forwards copies to interested observers. The central message log server is a CORBA object, and there can be multiple instances if warranted to support load leveling. The message log persistent store can be either a disk file or a database. The interested observers are primarily GUI windows on the screens of operators' consoles. While message logs are saved, observations are available in real-time as the messages are received. The framework allows any number of observers to be attached or detached at any time. 
Message attributes consist of the subsystem name, affected NIF component name, and time stamp. For real-time observers, these attributes allow filtering so that each window can be configured to display only those messages of interest at a particular console. The same attributes also allow selective retrieval of messages from saved logs.

Another responsibility of this framework is the alert system. Any ICCS application encountering a situation that requires immediate attention raises an alert, which then requires interaction with an operator to proceed. The alert system records its transactions so that the data can be analyzed after the fact.

\subsection{Reservations}

The reservation framework manages access to devices by giving one client exclusive rights to control or otherwise alter the device. The framework uses a lock-and-key model. Reserved devices that are "locked" can only be manipulated if and when a client presents the "key". Provision is made to allow an entire subtree from the configuration hierarchy to be reserved in one operation. Additionally, provision is made to construct groups of devices for convenient reservation. Read access to obtain status is not affected by the reservation.

As an example, the reservation service coupled with sequence control is typically used to implement machine process interlocks. These are specific configurations of the laser- and target-area hardware imposed to prevent damage to equipment (not related to personnel safety) or loss of shot data. Sequence control reserves needed devices and executes functions using the devices to establish conditions needed. Further access is prevented by the reservation service until the devices are released.

\subsection{System Manager}

The system manager provides services essential for the integrated management of the ICCS network of hundreds of computers. This component ensures necessary processes and computers are operating and communicating. Services include parameterized system startup, shutdown, and process watchdog monitoring. An important responsibility is coordinating the sequence of restarting portions (or all) of the ICCS computer system. FEPs can be started in either networked or stand-alone modes.

System manager is distributed; a central system manager expects periodic reports from system managers located in each workstation and FEP as to the operating status of the processes on each machine. The central system manager maintains a central view the entire ICCS and also collects metrics from the computers and network. This data may be reviewed to evaluate overall ICCS performance. Clients subscribe to the central system manager in order to be notified when a given processor or process fails or changes in a significant way.

\subsection{Machine History Archive}

Machine history is information about the performance and operation of the NIF that originates within the ICCS. The history archive collects data for analysis of the NIF operation in order to improve efficiency and reliability. Some information is also copied to the facility product data management (PDM) system to support the reliability, availability, and maintainability (RAM) goals of the facility. 
Examples of such information are:

- Installation and service of components

- Occurrences of abnormal conditions

- Records of operating service time or usage count

- Periodic readings of facility and environmental monitoring sensors

- Alignment reference images

- Experimental setup configurations

This framework allows different applications to define history records as extensible objects and to build histories as constantly growing series of history records. Any ICCS component can instantiate services from the history archive framework. History records are extensible to allow more attributes to be included in later history records as requirements evolve. The framework supports the retrieval of multiple histories of large groups of objects so that, for example, one can generate a report on the performance of all motors for studying reliability and predicting preventive maintenance.

\subsection{Generic Front End Processor}

The generic FEP framework pulls together the distributed aspects of the other frameworks (in particular system manager, configuration, status monitor, and reservation) by adding unique classes for supporting device and controller interfacing. These classes are responsible for hooking in CORBA distribution as well as implementing the creation, initialization, and connection of device and VO controller objects. The generic FEP also defines a common hardware basis including the target processor architecture, backplane, I/O boards, device drivers, and field bus support. The FEP application developer extends the base classes to incorporate specific functionality and state machine controls.

\subsection{Shot Data Archive}

The ICCS is not responsible for the permanent storage (archive) or in-depth study of shot data; it is however responsible for collecting the data from the diagnostics, making the data. immediately available for "quick look" analysis, and delivering the data to an archive. The framework contains a server working in collaboration with the system manager to assure that requested shot data are delivered to a disk staging area. The archive server is responsible for building a table of contents file and then forwarding the table and all data files to the archive.

An important aspect of this framework is that all data files will be written in one of a few approved formats. In addition to text files, the most favored format will be HDF files. Many commercial off-the-shelf visualization tools (such as Interactive Data Language) can read this self-describing data format, so the framework saves physicists from having to translate data formats before viewing their results. The definition of shot data includes not only the target diagnostics data but also timing and laser diagnostics data that will help experimentalists interpret their results.

\section{Software Deployment}

Many factors are considered during the object-oriented design process including the distribution and/or co-location of software objects, persistence, concurrency, and the 
partitioning of the system to optimize performance and minimize unnecessary coupling. In the deployment phase, objects are collected into run-time processes that execute on the ICCS computers. Objects instantiated from the framework classes, along with specialized objects created for the specific application, are coalesced into processes and allocated to computers in the network (Figure 7). Classes of computers in the system (diagrammed as shaded boxes) are the console workstation, the front end processor, and the file server. Devices that attach to the computers include the operator displays, databases, and control points. Entities listed inside the computer boxes represent the processes running under UNIX or, in the case of VxWorks, separate tasks.

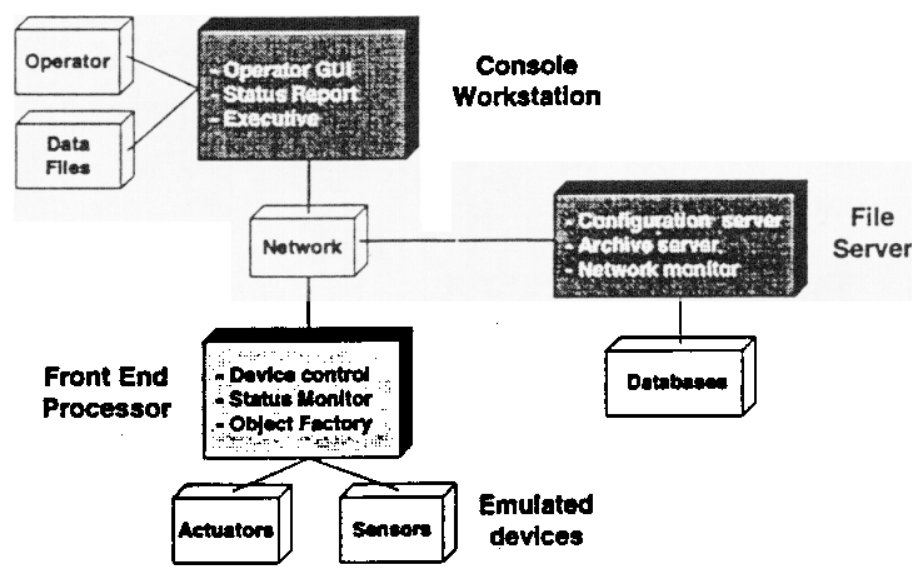

Figure 8 Deployment model shows processes allocated to system hardware

Main processes include the operator GUI, which is responsible for managing graphical output and operator input; many copies of this process can run to support more than one operator connection to the control system orchestrated by a common executive process. The status report process responds to many status monitor tasks (one runs on each front end processor) to collect and filter status changes in the equipment..The data collected is available to all interested broad-view status displays (managed by the operator GUI). The configuration server is utilized to startup control points in the FEP by collaborating with object factory tasks. During normal operation, all processes in the ICCS locate the CORBA references to device control tasks operating in the FEPs by using configuration services. The archive servers, as well as the configuration server, store and access persistent data managed by the database.

The performance and capacity of various ICCS deployment strategies is assessed by modeling and simulation, and verified by prototype measurements. In the event a shortcoming is detected, the architecture can easily be repartitioned or the network enhanced with faster equipment to meet requirements.

\section{Summary}

The ICCS is being developed using the iterative approach to software construction ${ }^{6}$. This technique is proven effective for projects whose requirements are not fully known until late in the project development. Five iterations are planned prior to the first facility deployment of the 
ICCS software. Each new release will follow a plan aimed at addressing the greatest risks to the architecture.

At this early stage in the ICCS design, the first prototype release has been completed and demonstrated on the ICCS testbed shown in Figure 9. The testbed includes several FEPs, a small tabletop alignment system, and an ATM network. It demonstrates integrated operation of six of the frameworks collaborating over CORBA communications.

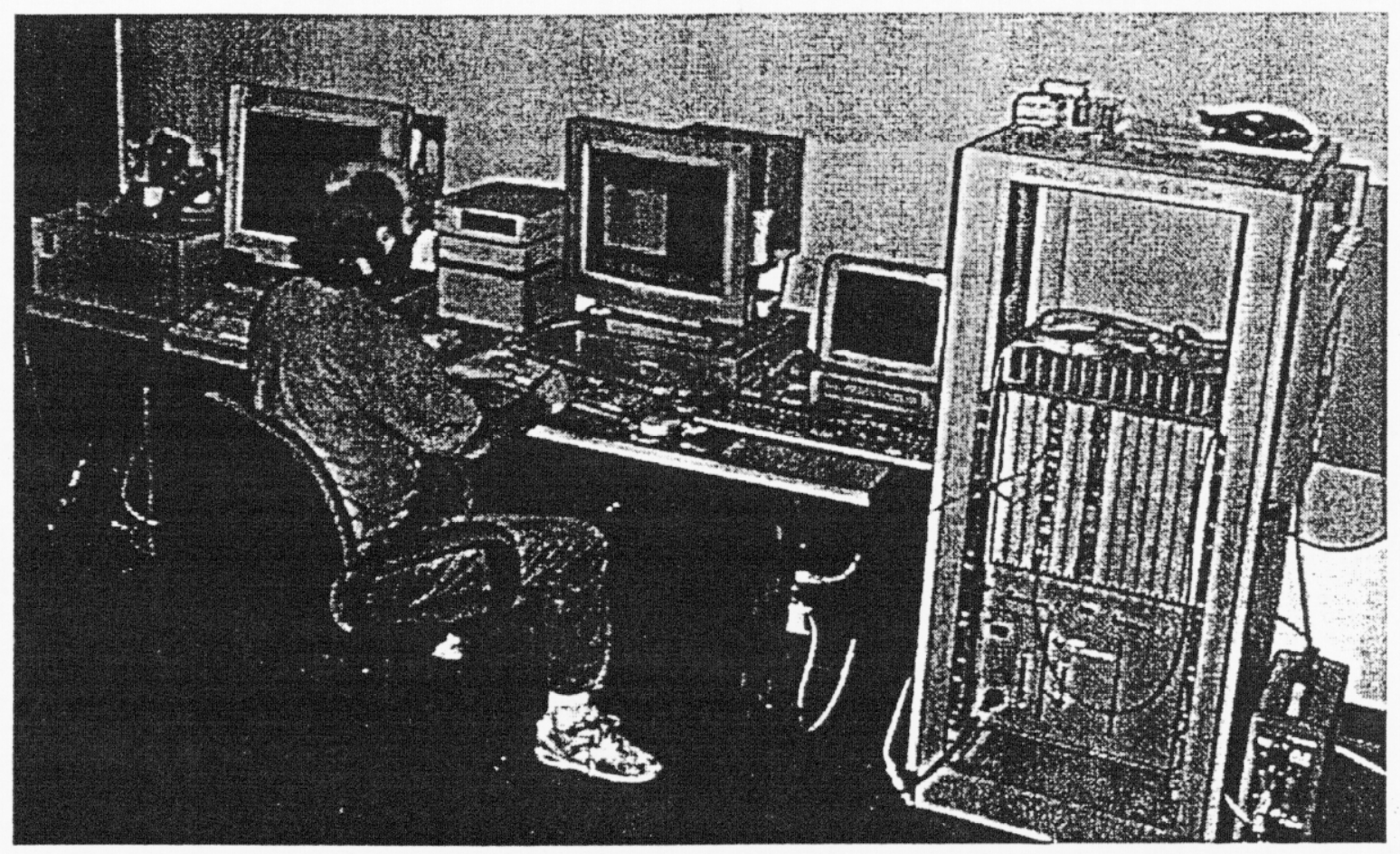

Figure 9 Integrated computer control system testbed

Construction of the ICCS incorporates many of the latest advances in computer and software technology. Primary goals of the design are to provide an open, extensible, and reliable architecture that can be maintained and upgraded for decades. The design permits software reuse and allows the system to be constructed within budget. The design is sufficiently abstract to allow future control systems to take advantage of this work. 


\section{References}

1. "ICCS Glossary of Terms", NIF-760

2. "Engineering Analysis and Literature Review of the use of CORBA in Distributed Object-Oriented Systems", NIF-314 (revised June 1997)

3. "Software Quality Assurance Plan for the National Ignition Facility Integrated Computer Control System", NIF-288

4. G. Booch, "Object-Oriented Analysis and Design", Benjamin/Cummings Publishing Company, Inc., Second Edition 1994

5. V. Swaminathan and J. Storey, "Domain-Specific Frameworks", Object Magazine, April 1988, pp. 53-57

6. "ICCS Device Naming Convention", NIF-2435

7. B. Boehm, "A Spiral Model of Software Development and Enhancement", IEEE Computer, May 1988, pp. 61-72 


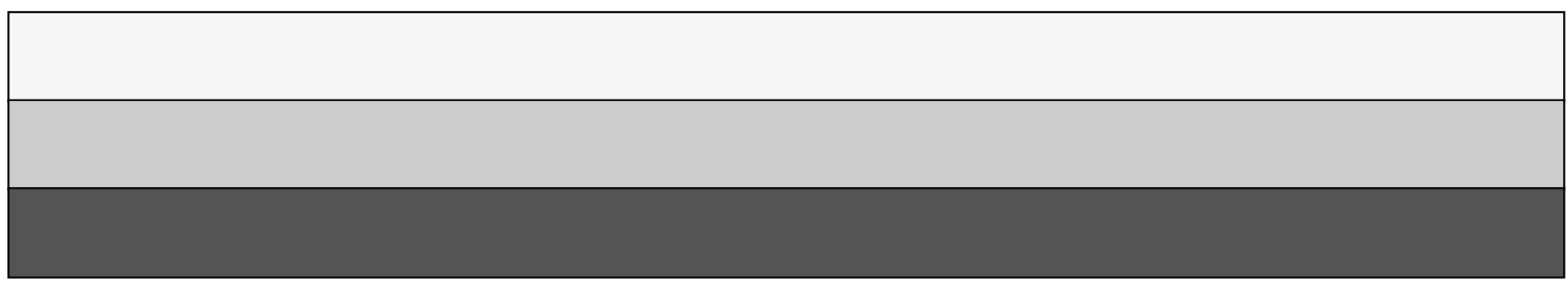

